\title{
Difference in blood pressure readings with mercury and automated devices: impact on hypertension prevalence estimates in Dar es Salaam, Tanzania
}

\author{
Arnaud Chiolero ${ }^{1}$, Jean-Pierre Gervasoni ${ }^{1}$, Anne Rwebogora ${ }^{2}$, Marianna Balampama ${ }^{2}$, Fred \\ Paccaud $^{1}$ \& Pascal Bovet ${ }^{1}$ \\ ${ }^{1}$ Institute of Social and Preventive Medicine, University of Lausanne, Lausanne, Switzerland; ${ }^{2}$ Ministry of Regional Admin- \\ istration and Local Government, Dar es Salaam, Tanzania
}

Accepted in revised form 4 April 2006

\begin{abstract}
Objectives: (1) To compare blood pressure (BP) readings with an automated arm cuff oscillometric device (AutoBP) to readings with a mercury sphygmomanometer $(\mathrm{HgBP})$ and (2) to evaluate the impact on the prevalence of hypertension (HBP) in a population-based survey. Methods: (1) In a convenience sample ("Comparison Study"), we measured BP with both AutoBP (Visomat ${ }^{\circledR}$ OZ2) and $\mathrm{HgBP}$ and we modeled $\mathrm{BP}$ difference $(\Delta \mathrm{BP}=\mathrm{HgBP}-\mathrm{AutoBP})$ with multiple regression analysis. (2) Using $\triangle \mathrm{BP}$, we calculated $\mathrm{HgBP}$ in a survey previously conducted in Dar es Salaam ("Population Survey") in which BP was measured with the automatic device Visomat ${ }^{\circledR} \mathrm{OZ} 2$ and we compared the prevalence of HBP $(\geq 140 / 90 \mathrm{mmHg}$ or
\end{abstract}

treatment). Results: In the Comparison Study (404 subjects aged 25-64), systolic/diastolic BP was higher by $4.4 / 4.7 \mathrm{mmHg}$ (SE: $0.4 / 0.3$ ) with $\mathrm{HgBP}$ than AutoBP. The prevalence of HBP was $42 \%$ with $\mathrm{HgBP}$ and $36 \%$ with AutoBP (relative difference of $14 \%) . \triangle \mathrm{BP}$ was associated with age, $\mathrm{BP}$ and arm circumference. In the Population Survey (9.254 subjects aged 25-64), the prevalence of HBP was $17 \%$ with calculated $\mathrm{HgBP}$ and $14 \%$ with AutoBP (relative difference of 20\%). Conclusion: A small systematic bias in BP readings between two different devices had large impact on hypertension prevalence estimates. This suggests that automated devices used in epidemiological studies should be validated with particular care.

Key words: Blood pressure measurement, Hypertension, Tanzania, Validation protocol

\section{Introduction}

The auscultatory technique with a mercury sphygmomanometer has long been considered as a good standard to measure blood pressure (BP) [1]. However, automated devices are used increasingly often in clinical practice and in epidemiological studies because such devices prevent several observer-related biases [2], operators need less training, and measurement procedures can be better standardized [1, 3-6]. In addition, the use of mercury devices is declining due to environmental reasons. Accordingly, the use of "validated and affordable" automated devices in low resources setting has been advocated by a WHO expert panel [7].

$\mathrm{BP}$ readings with automatic devices rely on built-in electronic algorithms and several standard validation protocols have been developed [8-11]. The reliability of automated devices can vary differentially over the range of various characteristics, such as BP or arm circumference, as shown by us [12] and others [5, 13-15]. Hence, validation protocols recommend that devices should be tested over a large range of BP [9, $11]$, in various age categories $[9,16]$, and on persons with small, medium and large arm circumference [8, 16]. Current validation protocols are not particularly stringent and may allow for significant BP differences between devices [17, 18]. While a small bias in BP readings from a given device may have limited significance at an individual level, it may have large impact when assessing the prevalence of hypertension (HBP) in epidemiological studies [17, 19]. Biases in $\mathrm{BP}$ readings may prevent valid comparison of $\mathrm{HBP}$ estimates between and within populations and mislead health planning.

In 1999, we assessed the prevalence of HBP in the population of Dar es Salaam, Tanzania [20] using a validated automated device [13]. We found a lower prevalence of HBP than another population-based survey that had used mercury sphygmomanometers [21]. We were unable to objectively quantify whether the difference could relate, at least in part, to the types of devices used in the surveys as we did not use a mercury device in our survey.

We subsequently conducted a study in an independent sample of subjects to compare BP readings between the automated device used in the survey and a mercury sphygmomanometer. We analytically 
modeled the BP difference between the devices and calculated BP based on a mercury sphygmomanometer that would have been obtained in the population survey and compared BP categories based on both devices.

\section{Methods \\ Comparison study}

We compared BP readings with a standard mercury sphygmomanometer to reading with a validated armcuff automated device (Visomat ${ }^{\circledR}$ OZ2) [13] in a convenience sample of apparently healthy adults visiting relatives or working at a hospital in Dar es Salaam. Four hundred and four subjects agreed to participate (referred hereafter as "Comparison Study").

$\mathrm{BP}$ was measured in the sitting position, after a rest of at least $5 \mathrm{~min}$, on the left arm, at 1-min intervals and at $2-\mathrm{mmHg}$ precision. Mid arm circumference was measured at $0.5 \mathrm{~cm}$ precision and cuff size was selected accordingly. Three readings were obtained with each device. Mercury and automated devices were used alternatively and the order in which the first device was chosen was selected randomly to avoid a systemic bias related to spontaneously decreasing BP over repeated readings [22]. With the mercury device, systolic and diastolic BP was measured at Korotkoff phases I and V. Measurements were conducted by trained clinical officers. Officers who took mercury readings were blind to results with the automated device, and inversely. The average of the second and the third $\mathrm{BP}$ readings with each device was used to define automated device BP (AutoBP) and mercury sphygmomanometer $\mathrm{BP}(\mathrm{HgBP})$, respectively. Difference in BP were calculated as $\Delta \mathrm{BP}=\mathrm{HgBP}-$ AutoBP.

\section{Population survey}

Between November 1998 and August 1999, we conducted a cross-sectional survey of cardiovascular risk factors in all adults living in five branches of the 710.000 inhabitants municipality of Temeke in Dar es Salaam, Tanzania (referred hereafter as "Population Survey"). Methods of the population survey, participants' characteristics and the distribution of BP and other risk factors have been reported previously [20]. The survey included 9254 subjects aged 25-64.

In the population survey, BP was measured at the participants' household, on persons seated, after a rest of at least $5 \mathrm{~min}$, on the left arm. Mid arm circumference was measured at $0.5 \mathrm{~cm}$ precision and cuff-size was selected accordingly. BP was measured with an arm-cuff oscillometric automated device Visomat ${ }^{\circledR}$ OZ2 (same device as used in the Comparison Study) [13]. Three BP readings were obtained at 1-min intervals. The average of the second and the third $\mathrm{BP}$ readings was used for $\mathrm{BP}$ estimates.

For both the Comparison Study and the Population Survey, subjects were free to participate and gave informed consent. Both studies were approved by the Tanzanian National Institute of Medical Research and the Tanzanian Commission for Science and Technology. Cut-off values for BP categories followed usual categories [23] and hypertension (HBP) was defined as $\mathrm{BP} \geq 140 / 90 \mathrm{mmHg}$ or current antihypertension treatment.

\section{Statistical analysis}

One way ANOVA and $t$-test were performed for comparison between continuous variables. BlandAltman plots were generated and limits of agreements between readings of systolic and diastolic BP with both devices were calculated [24]. With data from the Comparison Study, we generated separate multivariate linear regression models to predict systolic and diastolic $\Delta \mathrm{BP}$ that included, as independent variables age, sex, mid-arm circumference and BP level. In these models, we used categorical values (dummy variables) for the dependent continuous variables. We used these multivariate parameters to calculate $\mathrm{HgBP}$ of the participants to the Population Survey. We then compared the prevalence of BP categories based on AutoBP (measured) and $\mathrm{HgBP}$ (calculated) in the Population Survey. Age-standardized prevalence was adjusted to the age distribution of the world standard population proposed by the WHO [25]. Analyses were performed with Stata 8.0.

\section{Results}

Characteristics of the participants to the Comparison Study are presented in Table 1. Four hundred and four subjects (201 men, 203 women) participated in the study. Systolic and diastolic BP were higher with $\mathrm{HgBP}$ than with AutoBP. For systolic BP, mean $\triangle \mathrm{BP}$ was $4.4 \mathrm{mmHg}$ (standard error: 0.4 ) and limits of agreement ranged from -13.4 to $22.2 \mathrm{mmHg}$. For diastolic $\mathrm{BP}$, mean $\triangle \mathrm{BP}$ was $4.7 \mathrm{mmHg}(0.3)$ and limits of agreement ranged from -9.2 to $18.6 \mathrm{mmHg}$. Comparing $\mathrm{HgBP}$ to AutoBP, the prevalence of HBP $(\geq 140 / 90 \mathrm{mmHg}$ or treatment) was respectively $42.1 \%$ (95\% CI: $37.2-47.1)$ and $36.1 \%$ (31.4-41.0), a relative underestimation of $14 \%$, with AutoBP vs. $\mathrm{HgBP}$.

Table 2 shows $\triangle \mathrm{BP}$ across categories of selected variables in the Comparison Study. Systolic $\Delta \mathrm{BP}$ did not differ across sex and age categories but decreased with increasing categories of mid-arm circumference. Systolic $\Delta \mathrm{BP}$ was lower for the BP category $140-159$ / 90-99 mmHg. Diastolic $\Delta B P$ did not differ across sex categories but decreased with increasing categories of age, mid-arm circumference, and BP. 
Table 1. Characteristics of the participants to the Comparison Study

\begin{tabular}{lcccr}
\hline & Men $(\mathrm{N}=201)$ & SD & Women $(\mathrm{N}=203)$ & SD \\
\hline Age (years) & 40.6 & 11.6 & 41.1 & 11.8 \\
Body mass index $\left(\mathrm{kg} / \mathrm{m}^{2}\right)$ & 24.0 & 5.4 & 25.6 & 4.1 \\
Mid arm circumference $(\mathrm{cm})$ & 27.9 & 4.2 & 28.0 & 3.0 \\
Systolic HgBP $(\mathrm{mmHg})$ & 133.5 & 25.4 & 129.7 & 24.4 \\
Diastolic HgBP $(\mathrm{mmHg})$ & 87.1 & 15.6 & 85.8 & 14.6 \\
Systolic AutoBP $(\mathrm{mmHg})$ & $128.5^{* * *}$ & 24.8 & $126.0^{* * *}$ & 25.2 \\
Diastolic AutoBP $(\mathrm{mmHg})$ & $82.4^{* * *}$ & 18.2 & $81.1^{* * *}$ & 17.0 \\
\hline
\end{tabular}

Mean and standard deviation (SD).

$\mathrm{HgBP}$, blood pressure measured with the mercury sphygmomanometer.

AutoBP, blood pressure measured with the automated device.

$* * * p<0.001$ comparing readings with either device.

Table 3 shows the multivariate predictors of $\Delta \mathrm{BP}$ in the Comparison Study. The direction and magnitude of the associations were similar to those found in univariate analysis.

Based on the parameters from the multivariate analysis to predict $\triangle \mathrm{BP}$ (Table 3 ), we calculated $\mathrm{HgBP}$ in the Population Survey, i.e. BP that would have occurred if a mercury sphygmomanometer had been used for measurement instead of the automatic device. Table 4 shows that BP was substantially higher when readings were based on the mercury

Table 2. Difference in blood pressure measured with the mercury sphygmomanometer and the automated device $(\triangle \mathrm{BP})$ across selected characteristics of the subjects in the Comparison Study

\begin{tabular}{|c|c|c|c|c|c|c|}
\hline & $\mathrm{N}$ & $\begin{array}{l}\text { Systolic } \\
\Delta \mathrm{BP} \\
(\mathrm{mmHg})\end{array}$ & SE & $p$ & $\begin{array}{l}\text { Diastolic } \\
\Delta \mathrm{BP} \\
(\mathrm{mmHg})\end{array}$ & $\mathrm{SE} p$ \\
\hline \multicolumn{7}{|l|}{ Sex } \\
\hline Men & 201 & 5.0 & 0.6 & & 4.7 & 0.5 \\
\hline Women & 203 & 3.7 & 0.6 & ns & 4.7 & $0.5 \mathrm{~ns}$ \\
\hline \multicolumn{7}{|l|}{ Age (years) } \\
\hline $25-34$ & 150 & 5.0 & 0.6 & & 6.8 & 0.5 \\
\hline $35-44$ & 104 & 4.0 & 0.9 & & 5.2 & 0.7 \\
\hline $45-54$ & 79 & 2.9 & 1.1 & & 2.8 & 0.9 \\
\hline $55-64$ & 71 & 5.2 & 1.3 & ns & 1.8 & $0.7 * * *$ \\
\hline \multicolumn{7}{|c|}{ Mid arm circumference $(\mathrm{cm})$} \\
\hline$<27$ & 151 & 6.2 & 0.6 & & 5.9 & 0.5 \\
\hline $27-29$ & 131 & 4.6 & 0.8 & & 4.6 & 0.6 \\
\hline$\geq 30$ & 122 & 1.9 & 0.8 & $* * *$ & 3.3 & $0.7 * *$ \\
\hline \multicolumn{7}{|c|}{ Blood pressure category (mmHg) } \\
\hline$<120 / 80$ & 166 & 6.8 & 0.5 & & 8.0 & 0.4 \\
\hline $120-139 / 80-89$ & 106 & 3.6 & 0.8 & & 4.8 & 0.6 \\
\hline $140-159 / 90-99$ & 67 & 0.3 & 1.2 & & 2.0 & 0.8 \\
\hline$\geq 160 / 100$ & 65 & 3.5 & 1.4 & $* * *$ & -1.0 & $0.9 * * *$ \\
\hline
\end{tabular}

Mean and standard error (SE).

$\mathrm{BP}$ category based on BP readings with the automated device.

$\Delta \mathrm{BP}$, difference in blood pressure with the mercury sphygmomanometer and with the automated device. $* * p<0.01 ; * * * p<0.001$ for difference in $\Delta \mathrm{BP}$ between categories. device as compared to the automatic device in all age and sex categories in the Population Survey.

Table 5 shows that the prevalence of HBP (BP $\geq 140 / 90 \mathrm{mmHg}$ or treatment) was substantially underestimated in the Population Survey based on the automated device (measured) as compared to the mercury device (calculated). Comparing $\mathrm{HgBP}$ to AutoBP, the crude prevalence of HBP was $17.3 \%$ (16.5-18.0) vs. $13.8 \%$ (13.1-14.5), i.e. an underestimation of $20 \%$ with AutoBP vs. HgBP. The prevalence estimates were respectively $16.4 \%(15.4-17.4)$ vs. $13.2 \%(12.3-14.0)$ in men and $18.6 \%(17.3-19.9)$ vs. $14.9 \%(13.7-16.1)$ in women.

The prevalence of selected BP categories in the Population Survey differed markedly depending on the type of device used for assessing BP (Figure 1). A higher prevalence of the BP category $<120 / 80 \mathrm{mmHg}$ was observed with the automated device. For the other BP categories, lower proportions were observed with the automated device, but the magnitude of the difference tended to decrease with higher BP categories.

\section{Discussion}

Our data indicate that the automated device markedly underestimated $\mathrm{BP}$, as compared to measurements based on a mercury device. Consequently, in a population-based survey, the prevalence of HBP was underestimated by $20 \%$ with the automated device as compared to the mercury device.

The BP estimates in the population survey adjusted for the bias between the automatic and mercury devices are likely to better reflect the true levels in the population of Dar Es Salaam (assuming that the mercury sphygmomanometer constitutes the standard for BP measurement). This claim is also indirectly supported by the fact that the adjusted estimates better correspond to the prevalence found in another study in the same city in 1996 based on measurements with a mercury sphygmomanometer [21]. However, difference in the prevalence between the two studies could also relate to the fact that BP 
Table 3. Association of the difference in blood pressure between devices $(\Delta \mathrm{BP})$ with selected characteristics in the Comparison Study

\begin{tabular}{|c|c|c|c|c|c|c|}
\hline & \multicolumn{3}{|c|}{ Systolic $\Delta \mathrm{BP}(\mathrm{mmHg})$} & \multicolumn{3}{|c|}{ Diastolic $\Delta \mathrm{BP}(\mathrm{mmHg})$} \\
\hline & $\begin{array}{l}\text { Regression } \\
\text { coefficient }\end{array}$ & SE & $p$ & $\begin{array}{l}\text { Regression } \\
\text { coefficient }\end{array}$ & SE & $p$ \\
\hline \multicolumn{7}{|c|}{ Mid arm circumference $(\mathrm{cm})$} \\
\hline$<27$ & 0 & - & - & 0 & - & - \\
\hline $27-29$ & -0.9 & 1.0 & 0.091 & -1.2 & 0.7 & ns \\
\hline$\geq 30$ & -2.8 & 1.1 & 0.001 & -1.6 & 0.8 & 0.041 \\
\hline \multicolumn{7}{|l|}{ Age (years) } \\
\hline $25-34$ & 0 & - & - & 0 & - & - \\
\hline $35-44$ & 1.0 & 1.1 & ns & 0.6 & 0.8 & ns \\
\hline $45-54$ & 0.2 & 1.3 & ns & -1.0 & 0.9 & ns \\
\hline $55-64$ & 2.5 & 1.3 & ns & -2.3 & 0.9 & 0.016 \\
\hline \multicolumn{7}{|c|}{ Blood pressure category (mmHg) } \\
\hline$<120 / 80$ & 0 & - & - & 0 & - & - \\
\hline $120-139 / 80-89$ & -3.1 & 1.1 & 0.006 & -2.6 & 0.8 & 0.001 \\
\hline $140-159 / 90-99$ & -6.3 & 1.3 & 0.000 & -5.0 & 1.0 & 0.000 \\
\hline$\geq 160 / 100$ & -3.5 & 1.3 & 0.009 & -8.3 & 1.0 & 0.000 \\
\hline Constant & 7.2 & 0.9 & 0.000 & 8.9 & 0.6 & 0.000 \\
\hline
\end{tabular}

SE: standard error.

Blood pressure categories are based on the readings with the automated device.

$\Delta \mathrm{BP}$, difference in blood pressure with the mercury sphygmomanometer and with the automated device.

was based on the average of the second and third of three BP readings in our survey versus the average of the first and second readings in the survey by Edwards et al. [21].

In our comparison study, the arm circumference and $\mathrm{BP}$ level were the main predictors of the difference in $\mathrm{BP}(\triangle \mathrm{BP}) . \Delta \mathrm{BP}$ was not related in a linear manner but, instead, a difference was most apparent for selected ranges of $\mathrm{BP}$ and arm circumference. This is consistent with findings in several other studies that
$\Delta \mathrm{BP}$ cannot be easily anticipated or predicted. In a previously published validation study of the Visomat OZ2 ${ }^{\circledR}[13]$, systolic $\Delta \mathrm{BP}$ was higher in hypertensive compared to normotensive subjects, whereas diastolic $\Delta \mathrm{BP}$ was lower in hypertensive compared to normotensive subjects. In a comparison study of an automated device with an aneroid sphygmomanometer, Kroke et al. [5] found that systolic $\triangle \mathrm{BP}$ was associated with lean body mass and age in both sex, whereas diastolic $\triangle \mathrm{BP}$ was associated with age in

Table 4. Mean blood pressure $(\mathrm{mmHg})$ by age, sex and type of device in the Population Survey

\begin{tabular}{|c|c|c|c|c|c|c|}
\hline Age (years): & $25-34$ & $35-44$ & $45-54$ & $55-64$ & $25-64$ & $35-64 *$ \\
\hline \multicolumn{7}{|l|}{ Men } \\
\hline $\mathrm{N}$ & 1930 & 910 & 481 & 279 & 3600 & 1670 \\
\hline \multicolumn{7}{|l|}{ Systolic } \\
\hline AutoBP & $116.8(0.3)$ & $121.2(0.6)$ & $128.3(0.9)$ & $135.6(1.4)$ & $120.9(0.3)$ & $127.5(0.6)$ \\
\hline $\mathrm{HgBP}$ & $121.5(0.3)$ & $126.0(0.5)$ & $131.6(0.9)$ & $140.9(1.3)$ & $125.5(0.3)$ & $131.9(0.5)$ \\
\hline \multicolumn{7}{|l|}{ Diastolic } \\
\hline AutoBP & $71.2(0.2)$ & $75.8(0.4)$ & $80.5(0.6)$ & $82.3(0.9)$ & $74.5(0.2)$ & $79.2(0.4)$ \\
\hline $\mathrm{HgBP}$ & $75.8(0.2)$ & $80.7(0.4)$ & $83.8(0.6)$ & $87.6(0.8)$ & $79.0(0.2)$ & $83.6(0.3)$ \\
\hline \multicolumn{7}{|l|}{ Women } \\
\hline $\mathrm{N}$ & 3601 & 1191 & 540 & 322 & 5654 & 2053 \\
\hline \multicolumn{7}{|l|}{ Systolic } \\
\hline AutoBP & $111.6(0.2)$ & $119.0(0.5)$ & $130.3(1.0)$ & 138.9 (1.4) & $116.5(0.3)$ & $127.7(0.6)$ \\
\hline $\mathrm{HgBP}$ & $116.8(0.2)$ & $124.0(0.5)$ & $133.5(1.0)$ & $144.0(1.3)$ & $121.5(0.2)$ & $132.2(0.3)$ \\
\hline \multicolumn{7}{|l|}{ Diastolic } \\
\hline AutoBP & $69.2(0.2)$ & $74.5(0.4)$ & $79.9(0.6)$ & $81.5(0.9)$ & $72.0(0.2)$ & $78.0(0.3)$ \\
\hline $\mathrm{HgBP}$ & $74.4(0.2)$ & $79.5(0.3)$ & $83.1(0.6)$ & $86.6(0.8)$ & $77.0(0.2)$ & $82.5(0.3)$ \\
\hline
\end{tabular}

$\mathrm{BP}$ values are expressed as mean (and standard error).

AutoBP, BP measured with the automated device.

$\mathrm{HgBP}, \mathrm{BP}$ corresponding to measurements with a mercury device calculated with parameters in Table 3 .

* BP adjusted for age to the WHO world standard population [25]. 
Table 5. Prevalence of hypertension ( $\mathrm{BP} \geq 140 / 90 \mathrm{mmHg}$ and/or treatment) by sex, age and type of device in the Population Survey

\begin{tabular}{|c|c|c|c|c|c|c|}
\hline Age (year): & $25-34$ & $35-44$ & $45-54$ & $55-64$ & $25-64$ & $35-64 *$ \\
\hline \multicolumn{7}{|l|}{ Men } \\
\hline $\mathrm{N}$ & 1930 & 910 & 481 & 279 & 3600 & 1670 \\
\hline AutoBP \% & $6.7(0.6)$ & $14.9(1.2)$ & $29.1(2.1)$ & $47.0(3.0)$ & $13.2(0.4)$ & $28.2(1.2)$ \\
\hline $\mathrm{HgBP} \%$ & $9.5(0.7)$ & $20.4(1.3)$ & $30.8(2.1)$ & $54.5(3.0)$ & $16.4(0.5)$ & $32.8(1.2)$ \\
\hline \multicolumn{7}{|l|}{ Women } \\
\hline $\mathrm{N}$ & 3601 & 1191 & 540 & 322 & 5654 & 2053 \\
\hline AutoBP \% & $5.5(0.4)$ & $16.2(1.1)$ & $35.2(2.1)$ & $50.6(2.8)$ & $14.9(0.6)$ & $28.9(1.1)$ \\
\hline $\mathrm{HgBP} \%$ & $8.0(0.5)$ & $21.2(1.2)$ & $36.9(2.1)$ & $58.1(2.8)$ & $18.6(0.6)$ & $33.3(1.1)$ \\
\hline
\end{tabular}

Prevalence (and standard error).

AutoBP, BP measured with the automated device.

$\mathrm{HgBP}, \mathrm{BP}$ corresponding to measurements with a mercury device calculated with parameters in Table 3 .

*BP adjusted for age to the WHO world standard population [25].

men, and with upper arm circumference and BP level in women. It was suggested that overestimation of BP by a Dinamap oscillometric automated device could relate to increased arterial stiffness [26], which is typically found in elderly people. This bias is unlikely to have occurred in our study as age, a major determinant of arterial stiffness, was not associated with systolic BP difference, at least up to age 64 . However, age tended to be associated with diastolic BP difference.

The automated device that we used did satisfy the validation criteria of the Association for the
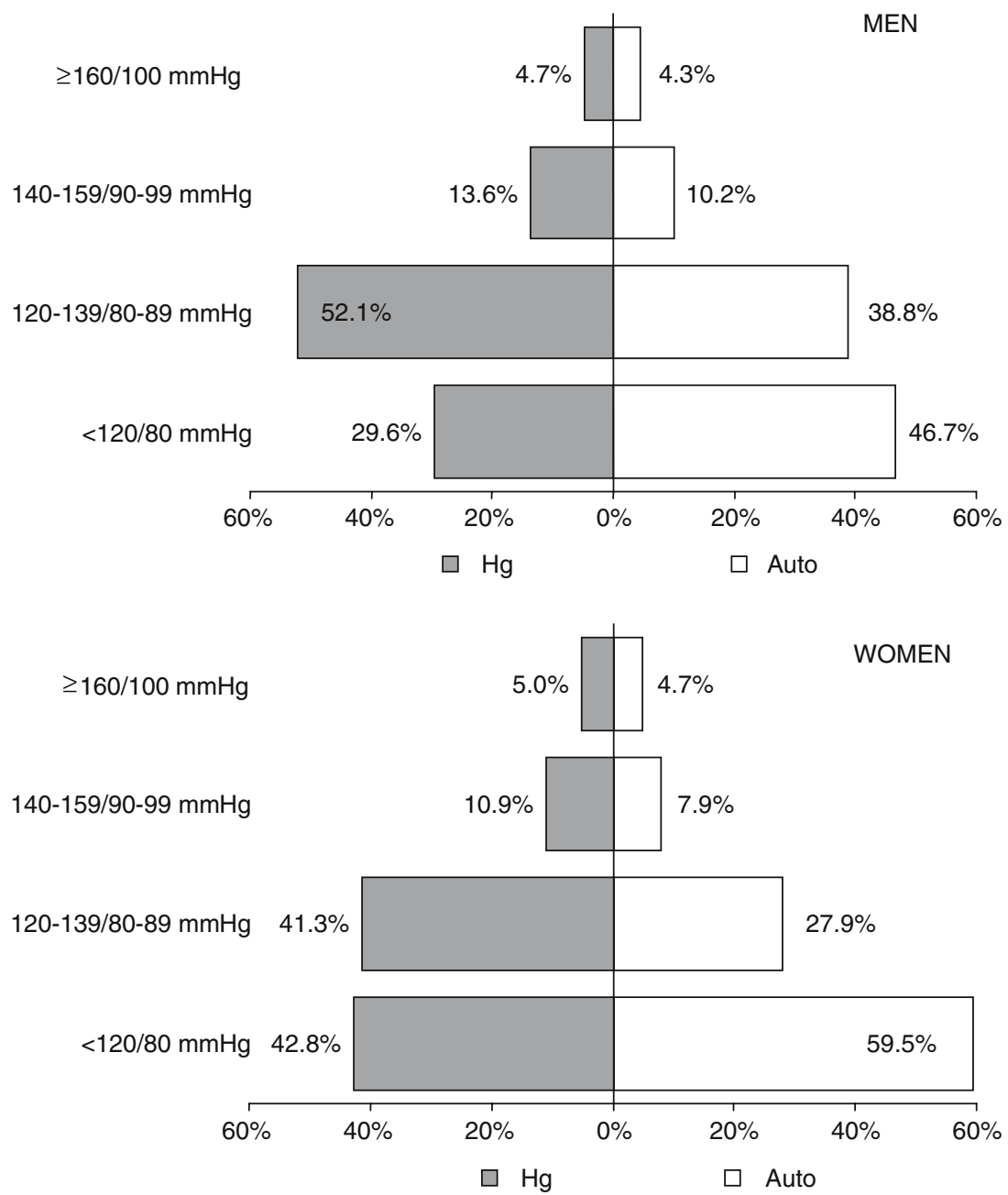

Figure 1. Prevalence of blood pressure categories based on readings with a mercury sphygmomanometer (HgBP; calculated) or an automated device BP (AutoBP; measured); men and women aged 25-64 years, Dar es Salaam, Tanzania. 
Advancement of Medical Instrumentation (AAMI) protocol $[8,13]$ and, partly, those of the British Hypertension Society (BHS) protocol $[9,13]$. In the validation study of this device [13], the mean difference in BP between the automated device and the mercury sphygmomanometer was $3.7 \pm 7.5 /$ $4.8 \pm 5.6 \mathrm{mmHg}$, which is consistent with our findings. The instrument used in our study is no longer marketed and is not recommended by the European Society of Hypertension [10]. However, our findings are likely to have relevance for other past, current, or future surveys of HBP that rely on validated automated devices.

A small bias in epidemiological studies can lead to large differences in the estimated prevalence of HBP in a population. In a study comparing $\mathrm{BP}$ readings with an aneroid device and readings with an automated device (BOSO Oscillomat ${ }^{\circledR}$ ), a systematic difference as small as $0.2 \pm 5.6 / 0.5 \pm 3.5 \mathrm{mmHg}$ led to miss one male hypertensive case out of 12 (HBP defined as $\mathrm{BP} \geq 160 / 95 \mathrm{mmHg}$ ) and one female case out of 50 [5]. More recently, it was found that an underestimation of systolic BP readings by $5 \mathrm{mmHg}$ could cause $21 \%-30 \%$ of patients with $\mathrm{BP} \geq 140$ / $90 \mathrm{mmHg}$ to be missed, whereas underestimation of diastolic BP by $5 \mathrm{mmHg}$ could cause respectively $47 \%-62 \%$ of hypertensive patients to be missed [19]. Based on the NHANES data, it was estimated that a difference of $1 \mathrm{mmHg}$ in $\mathrm{BP}$ would translate in a prevalence change of $2 \%$ [Richard Cooper, personal communication, May 2005]. Expectedly, the impact will largely depend on the distribution of BP in the population considered and will be greater if the population mean BP is close to the cut-off for HBP definition (e.g. 130-150/85-95 mmHg).

Systematic misclassification of HBP will be limited with clinically validated devices. However, current validation protocols do not ensure that such problems cannot occur with adequately validated automated devices [27]. Some authors stated that current validation protocols fail to ensure accuracy of automated devices [18, 27]. Indeed, several issues can be raised. For example, a $\mathrm{BP}$ monitor that meets the AAMI and BHS validation criteria may report BP in error by more than $5 \mathrm{mmHg}$ in more than $50 \%$ of the participants to validation studies, and by more than $10 \mathrm{mmHg}$ in more than $25 \%$ [17]. In addition, current validation protocols require the participation of a very low number of participants. Furthermore, while there are some requirements concerning the range of $\mathrm{BP}$ across which devices must be validated $[8,9]$, such requirements have been relaxed in international protocol to encourage manufacturers to submit BP automated device for validation [11].

While current validation protocols may possibly be sufficient for devices intended to be used at an individual level, a more stringent protocol might be considered for the validation of devices recommended for epidemiological studies. Such recommendations could include, for example, the need for a specified larger minimal total sample of subjects included in validation studies (e.g. $\geq 200$ ); a specified minimal number of persons with BP close to critical BP values for epidemiology (e.g. $\geq 100$ persons with BP of 130-150/85$95 \mathrm{mmHg}$ ); a specified maximal overall tolerance in measurements carried out in this critical BP range (e.g. $(\leq 1 \mathrm{mmHg}$ ); and a specified minimal number of persons with small, medium and large arms.

Pending more stringent validation protocols, investigators using automated devices in epidemiological studies could systematically validate automated devices within a subgroup of their study participants. Also, manufacturers could systematically provide a Y connection with all automated devices so that operators can easily compare BP readings given by the automated device to readings with a mercury device connected to the automated device by means of the $\mathrm{Y}$ tube.

In conclusion, a small bias in BP readings between different types of sphygmomanometers can have a large impact on hypertension prevalence estimates. Automated devices used in epidemiological studies should be validated with particular care, and possibly with specific validation protocols. Comparison studies should be replicated in different settings (e.g. other populations) and under different conditions (e.g. other devices) to further characterize the bias that can result from the use of different devices.

\section{Acknowledgements}

We thank Dr Deo Mtasiwa, City Medical Officer, Dar es Salaam, for support to cardiovascular epidemiological research and Prof Michel Burnier, University Hospital, Lausanne, for valuable comments on previous versions of the manuscript. Funding: Grant of the Swiss National Science Foundation (No 32-51189.97).

\section{References}

1. Pickering TG, Hall JE, Appel LJ, et al. Subcommittee of Professional and Public Education of the American Heart Association Council on High Blood Pressure Research. Recommendations for blood pressure measurement in humans and experimental animals: Part 1: blood pressure measurement in humans: a statement for professionals from the Subcommittee of Professional and Public Education of the American Heart Association Council on High Blood Pressure Research. Hypertension 2005; 45: 142-161.

2. Rose G. Standardisation of observers in blood pressure measurement. Lancet 1965; 10: 673-674.

3. Bruce NG, Shaper AG, Walker M, Wannamethee G. Observer bias in blood pressure studies. J Hypertens 1988; 6: 375-380. 
4. Cooper R, Puras A, Tracy J, et al. Evaluation of an electronic blood pressure device for epidemiologic studies. Blood Press Monit 1997; 2: 35-40.

5. Kroke A, Fleischhauer W, Mieke S, Klipstein-Grobusch $\mathrm{K}$, Willich SN, Boeing H. Blood pressure measurement in epidemiological studies: a comparative analysis of two methods. Data from the EPIC-Potsdam Study. European Prospective Investigation into Cancer and Nutrition. J Hypertens 1998; 16: 739-746.

6. Kaplan N. Measurement of blood pressure. In: Kaplan (ed.) Clinical Hypertension, 8th edn. Baltimore: Lippincott Williams \& Wilkins Publishers, 2002, p. 19-39.

7. Parati G, Mendis S, Abegunde D, et al. Recommendations for blood pressure measuring devices for office/ clinic use in low resource settings. Blood Press Monit 2005; 10: 3-10.

8. American National Standard. Electronic or Automated Sphygmomanometer. Arlington, VA: Association for the Advancement of Medical Instrumentation, 1993.

9. O'Brien E, Petrie J, Littler W, et al. An outline of the revised British Hypertension Society protocol for the evaluation of blood pressure measuring devices. J Hypertens 1993; 11: 677-679.

10. O'Brien E, Waeber B, Parati G, Staessen J, Myers MG. On the behalf of the European Society of Hypertension Working Group on Blood Pressure Monitoring. Blood pressure measuring devices: recommendations of the European Society of Hypertension. Br Med J 2001; 322: 531-536.

11. O’Brien E, Pickering T, Asmar R, et al. Working group on blood pressure monitoring of the European Society of Hypertension International Protocol for validation of blood pressure measuring devices in adults. Blood Press Monit 2002; 7: 3-17.

12. Chiolero A, Gervasoni JP, Rwebogora A, et al. Discordant prevalence of hypertension using two different automated blood pressure measurement devices: a population-based study in Dar es Salaam (Tanzania). Blood Press Monit 2004; 9: 59-64.

13. Dieterle T, Battegay E, Bucheli B, Martina B. Accuracy and ,range of uncertainty“ of oscillometric blood pressure monitors around the upper arm and the wrist. Blood Press Monit 1998; 3: 339-346.

14. Mee F, Atkins N, O'Brien E. Evaluation of the Profilomat II ambulatory blood pressure system according to the protocols of the British Hypertension Society and the Association for the Advancement of Medical Instrumentation. Blood Press Monit 1998; 3: 353-361.

15. Longo D, Bertolo O, Toffanin G, Frezza P, Palatini P. Validation of the A\&D UA-631 (UA-779 Life Source) device for self-measurement of blood pressure and relationship between its performance and large artery compliance. Blood Press Monit 2002; 7: 243-248.
16. O'Brien E, Atkins N. A comparison of the British Hypertension Society and Association for the Advancement of Medical Instrumentation protocols for validating blood pressure measuring devices: can the two be reconciled? J Hypertens 1994; 12: 1089-1094.

17. Gerin W, Schwartz AR, Schwartz JE, et al. Limitations of current validation protocols for home blood pressure monitors for individual patients. Blood Press Monit 2002; 7: 313-318.

18. Schwartz AR, Haas DC, Gerin W, Pickering TG. Accurate measurement of blood pressure. JAMA 2003; 289: 2792.

19. Turner MJ, Baker AB, Kam PC. Effects of systematic errors in blood pressure measurements on the diagnosis of hypertension. Blood Press Monit 2004; 9: 249-253.

20. Bovet P, Ross AG, Gervasoni JP, et al. Distribution of blood pressure, body mass index and smoking habits in the urban population of Dar es Salaam, Tanzania, and associations with socioeconomic status. Int $\mathbf{J}$ Epidemiol 2002; 31: 240-247.

21. Edwards R, Unwin N, Mugusi F, et al. Hypertension prevalence and care in an urban and rural area of Tanzania. J Hypertens 2000; 18: 145-152.

22. Bovet P, Gervasoni J-P, Ross AG, et al. Assessing the prevalence of hypertension in population: are we doing it right? J Hypertens 2003; 21: 509-517.

23. Joint National Committee on Prevention, Detection, Evaluation, and Treatment of High Blood Pressure. The Seventh Report of the Joint National Committee on Prevention, Detection, Evaluation, and Treatment of High Blood Pressure: the JNC 7 report. JAMA 2003; 289: 2560-2572.

24. Bland JM, Altman DG. Statistical methods for assessing agreement between two methods of clinical measurement. Lancet 1986; 1: 307-310.

25. Ahmad OB, Boschi-Pinto C, Lopez AD, Murray CJL, Lozano R, Inoue M. Age Standardisation of Rates: A New WHO World Standard. GPE Discussion Paper Series 31. Geneva: WHO, 2000.

26. van Popele NM, Bos WJ, de Beer NA, et al. Arterial stiffness as underlying mechanism of disagreement between an oscillometric blood pressure monitor and a sphygmomanometer. Hypertension 2000; 36(4): 484 488.

27. Pater C. Beyond the evidence of the new hypertension guidelines. Blood pressure measurement - is it good enough for accurate diagnosis of hypertension? Time might be in, for a paradigm shift (I). Curr Control Trials Cardiovasc Med 2005; 6: 6.

Address for correspondence: Pascal Bovet, Institute of Social and Preventive Medicine, University of Lausanne, 17, rue du Bugnon, 1005, Lausanne, Switzerland

Phone: + 41-21-3147272; Fax: +41-21-3147373

E-mail: pascal.bovet@chuv.ch 